Relations industrielles

Industrial Relations

\title{
Unité syndicale et cartels intersyndicaux
}

\section{Jean-Pierre Després}

Volume 3, numéro 7, mars 1948

URI : https://id.erudit.org/iderudit/1023606ar

DOI : https://doi.org/10.7202/1023606ar

Aller au sommaire du numéro

Éditeur(s)

Département des relations industrielles de l’Université Laval

ISSN

0034-379X (imprimé)

1703-8138 (numérique)

Découvrir la revue

Citer cet article

Després, J.-P. (1948). Unité syndicale et cartels intersyndicaux. Relations industrielles / Industrial Relations, 3(7), 106-107.

https://doi.org/10.7202/1023606ar

Tous droits réservés @ C Département des relations industrielles de l’Université Laval, 1948
Ce document est protégé par la loi sur le droit d'auteur. L'utilisation des services d'Érudit (y compris la reproduction) est assujettie à sa politique d'utilisation que vous pouvez consulter en ligne.

https://apropos.erudit.org/fr/usagers/politique-dutilisation/ 
«purged» them, under the pretext of «treason against Marxism », had not a merciful death in Victorian England saved them long ago from undesired martyrdom.

These two main discrepancies between the philosophy of the «Manifesto» and sucial reality cannot but gravely affect the strength of the main foundation upon which Marx' house is built. The Marx of the «Manifesto » has not the slightest doubt that the seizure of political power by the proletariat will be a revolutionary act. Later in his life, however, Marx himself no longer believed in this article of faith. He admitted that in certain advanced countries the victory of the proletariat may be achieved without revolutionary violence. But what does «victory of the proletariat » under these circumstances mean ? It can only signify that nembers of the proletariat will gradually ascend and infiltrate into the ruling stratum and obtain a share in government equal to the social importance of the industrial working class. This, once more, is only common sense; it would have needed no Karl Marx to show it. All analysts of human society, from Aristotle to Mosca, Sorel and Pareto, are united in this truth.

Indeed in the whole Western World, the mutual absorption of ruling class and working masses appears now to be under way «without revolutionary violence ». And it is rather disconcerting for the Marxists to observe that these are precisely the industrialized countries, such as England, where one could find some resemblance to the two-class-system of Marx and for which Marx doctrine of revolution was coined in the first place. The real social revolutions, on the other side, are taking place in the agrarian countries of the European East and of Asia. For them the «Manifesto » cannot mean a philosophy of history of an even moderate degree of precision, but at the best a quasi-religious and rather vague «fanal» to establish a minimum of social justice. In these countries Marx' ideas are now being applied to justify some sort of state-capitalism and to develop them according to the industrial pattern set by Western Europe and America. And to crown this mountain of inconsistency: in Russia and the whole Slav world, Communism is increasingly transformed into a violent panslavist nationalism which would only make Marx turn in his grave.

\section{UNITÉ SYNDICALE ET CARTELS INTERSYNDICAUX}

\section{Jean-Pierre DESPRES}

La reconnaissance du principe de la liberté syndicale est consacrée par la législation du travail aussi bien que par l'évolution des relations industrielles. La liberté syndicale implique le droit pour le salarié d'adhérer à une organisation de son choix. En principe, sauf dans certains cas précis, elle implique également le droit de n'adhérer à aucune organisation. "La divergence iảéologique, au sein des sociétés modernes, déclare une résolution de la Confédération internationale des syndicats chrétiens, implique des conceptions différentes au sujet de la vie présente et future, des droits et des devoirs personnels, familiaux, professionnels et sociaux. » C'est pourquoi un travailleur adhèrera à telle organisation syndicale de préférence à telle autre. Il en résulte plusieurs groupements syndicaux dans un même pays, par suite de cette divergence idéologique entre les divers groupes de salariés. En d'autres termes, le pluralisme syndical est la conséquence logique du principe de la liberté syndicale. Est-ce un mal, ou plus exactement un obstacle au développement du syndicalisme considéré dans son ensemble? Nier le droit au pluralisme syndical équivaut à s'attaquer à la liberté syndicale elle-même et conférer, en fait, une orientation totalitaire à l'action syndicale.

Il ne s'agit pas de nier les avantages de l'unité syndicale ou de fermer les yeux sur les inconvénients réels du pluralisme syndical. La réalisation de l'unité syndicale et le développement du pluralisme syndical résultent de circonstances qui varient selon les pays. Au Royaume-Uni, par exemple, le problème du pluralisme syndical ne s'est pas posé jusqu'à présent. 'Il en est différemment des Etats-Unis, du Canada, de la France, de ia Belgique, de la Suisse, etc., où le pluralisme syndical est la règle. A moins de verser dans le t'talitarisme, on ne saurait, en principe et en fait, contester la reconnaissance du pluralisme syndical.

$\mathrm{Ne}$ vaudrait-il pas mieux pour la classe ouvrière n'avoir qu'une seule organisation centrale dans chaque pays et une seule organisation sur le plan international ? L'unité syndicale ne confèrerait-elle pas une influence plus considérable auprès des pouvoirs publics et du patronat ? La ré- 
ponse à ces deux questions est affirmative. Sans aucun doute, l'unité syndicale, sur le plan dcs réalisations matérielles, présente une supériorité sur le pluralisme syndical. Mais lorsque l'unité syndicale ne découle pas de l'évolution volontaire et normale des organisations professionnelles de salariés, mais résulte de la pression d'un groupement majoritaire ou de celle de l'Etat, on assiste alors à une mutilation de la liberté, à une opération qui, si elle comporte des avantages immédiats, n'offre aucune garantie de permanence. Il ne manque pas d'exemples pour démontrer que l'unité syndicale artificielle est une plante fragile qui ne résiste pas à la première tourmente venue. Toutefois, la reconnaissance du pluralisme syndical, si elle vient à l'encontre de l'unité organique des groupements, n'est pas un obstacle à l'unité d'action des salariés. Si le pluralisme syndical découle de la liberté syndicale, l'unité de représentation devrait être le complément indispensable du pluralisme syndical. En d'autres termes, l'évolution des relations industrielles et la participation de plus en plus étendue des syndicats à l'activité économique et sociale exigent une attitude commune de l'ensemble du mouvement syndical devant le patronat et l'Etat. C'est par les cartels intersyndicaux, organes de représentation unique auprès des employeurs et de l'Etat, qu'il est possible de pallier aux inconvénients du pluralisme syndical.

L'idée du cartel intersyndical n'est pas nouvelle. Il faudrait remonter loin dans le passé pour en découvrir la première application. Que de fois des organisations syndicales d'inspiration différente ont constitué de fait un cartel intersyndical en soumettant des vues analogues sur tel ou tel projet de loi. La législation elle-même reconnaît le principe du cartel intersyndical, puisqu'elle permet à des organisations syndicales de grouper leurs effectifs en vue d'obtenir le statut d'agent négociateur dans une entreprise. Dans les comités paritaires, par exemple, la participation de deux ou trois syndicats à la négociation de la convention collective et à l'application du décret, équivaut à la formation implicite d'un cartel intersyndical.

N'y aurait-il pas lieu de doter le cartel intersyndical d'un statut institutionnel, étant donné l'absence d'unité syndicale parmi les travailleurs canadiens ? Jusqu'ici le cartel intersyndical a revêtu un caractère temporaire en raison de l'objectif qui lui a donné naissance: défendre les organisations de salariés contre une menace commune. En d'autres termes, le cartel intersyndical visait principalement des objectifs transitoires. Mais le cartel intersyndical - ou plus exactement la collaboration libre et rationnelle des organisations ouvrières entre elles - peut rendre des services autrement plus positifs et plus durables.

En France lorsqu'il fut question de constituer un comité interconfédéral d'cntente syndicale au mois de septembre 1944, ses attributions devaient être les suivantes: «a) désigner ou présenter les délégués au sein des différents organismes nationaux et internationaux interprofessionnels auxquels les confédérations ouvrières sont appelées à collaborer: b) rechercher l'accord entre les deux confédérations ouvrières sur les problèmes qui sc posent dans le domaine du social et de l'économique au sein des organismes nationaux et internationaux interprofessionnels; c) preparer, promou. voir et réaliser les grandes campagnes en faveur des revendications ouvrières, tant dans le cadre national que dans le cadre international; d) étudier ou faire mettre à l'étude par les fédérations ou unions de syndicats, toutes les questions rolatives au travail et aux conditions générales de vie des salariés; e) intervenir et se prononcer en dernier ressort, et après délibérations des unicns et fédérations compétentes, sur les litiges qui pourraient surgir entre organisations syndicales appartenant respectivement à l'une et l'autre des coufédérations; f) intervenir et formuler un avis au sujet du caractère représentatif des organisations syndicales, chaque fois qu'une difficulté surviendrait; g) échanger toute documentation utile à l'application du présent accord». Pour des raisons que nous n'avons pas à examiner ici, ce projet d'accord n'a pas eu de suite, mais cela n'empêche qu'il apportait un compromis entre le pluralisme syndical et la solidarité ouvrière. L'unité d'action, à défaut de l'unité syndicale, comble les déficiences du pluralisme syndical en harmonisant les « requêtes de l'unité avec les exigences de la liberté ».

La permanence du fait syndical, le développement de la législation du travail, la participation des organisations de salariés à l'activité économique et sociale dans le cadre de l'entreprise, dans le cadre de l'industrie et dans le cadre gouvernemental, la nécessité d'établir un ordre dans les relations industrielles en général, suggèrent naturellement la constitution d'un comité d'entente intersyndicale. 